\title{
A NEW MEASURE OF MORTALITY \\ DIFFERENTIALS BASED ON PRECEDENCE PROBABILITY
}

Cadena, M. and M. Denuit

\section{LIDAM Discussion Paper ISBA/2021/11}




\title{
A NEW MEASURE OF MORTALITY DIFFERENTIALS BASED ON PRECEDENCE PROBABILITY
}

\author{
MEITNER CADENA \\ Departamento de Ciencias Exactas - DECE \\ UFA-ESPE \\ Sangolqui, Ecuador \\ MICHEL DENUIT
}

Institute of Statistics, Biostatistics and Actuarial Science - ISBA

Louvain Institute of Data Analysis and Modeling - LIDAM

UCLouvain

Louvain-la-Neuve, Belgium

January 26, 2021 


\begin{abstract}
This short note aims to propose a new comparison measure for life tables: the age shift needed to restore equality of survival chance as measured by the precedence probability. Mortality by household income in France and by generation in Belgium is used as an illustration.
\end{abstract}

Key words and phrases: Stochastic precedence, life tables, mortality projection, mortality differentials. 


\section{Precedence probability}

Comparing the respective performance of competing systems is of particular interest in reliability analysis. Stochastic precedence has been proposed as an effective tool in that respect. This concept is based on the relevant probability $\mathrm{P}\left[T_{1} \leq T_{2}\right]$, where $T_{1}$ and $T_{2}$ denote the respective system lifetimes. Specifically, $T_{1}$ is said to stochastically precede $T_{2}$ when $\mathrm{P}\left[T_{1} \leq T_{2}\right] \geq 0.5$. This definition can be traced back (at least) to Brown and Rutemiller (1973). For continuous random variables $T_{1}$ and $T_{2}$, this holds true if, and only if, $\mathrm{P}\left[T_{1} \leq T_{2}\right] \geq \mathrm{P}\left[T_{1} \geq T_{2}\right]$ so that it is more likely that the first system has broken down while the second one was still working. If $T_{1}$ and $T_{2}$ are independent and identically distributed, we obviously have $\mathrm{P}\left[T_{1} \leq T_{2}\right]=0.5$.

The precedence probability $\mathrm{P}\left[T_{1} \leq T_{2}\right]$ allows the analyst to classify the second system as better than, equivalent to or worse than the first system according to whether this probability is greater than, equal to or less than 0.5. It is rooted in the history of nonparametric testing, corresponding to the expected value of the celebrated Mann-Whitney statistic used to test the equality of two distributions. This concept is relevant in numerous engineering applications, such as stress-strength for instance. It also naturally appears in the targetbased approach to decision under risk, as pointed out by De Santis, Fantozzi and Spizzichino (2015). Other references about stochastic precedence include Arcones, Kvam and Samaniego (2002), Boland, Singh and Cukic (2004), Navarro and Rubio (2010), and Finkelstein (2013).

The precedence probability can be used to compare life tables, considering independent remaining lifetimes $T_{x}^{[1]}$ and $T_{x}^{[2]}$ at attained age $x$ obeying each of them. If $\mathrm{P}\left[T_{x}^{[1]} \leq T_{x}^{[2]}\right] \geq$ 0.5 then the second life table is associated with better survival. Being based on outliving probabilities, stochastic precedence conforms with intuition. In this letter, we propose a related concept to compare life tables.

\section{Mortality differentials}

\subsection{Age shift restoring equality of survival chance}

Equality of chance (or opportunity) has become an important concept in public policy. Reaching a precedence probability $\mathrm{P}\left[T_{x}^{[1]} \leq T_{x}^{[2]}\right]=0.5$ for two individuals aged $x$ in a population of interest is thus appealing in that respect since it ensures that none of them is more likely to survive to the other. When the two individuals are taken from different socio-economic groups, the precedence probability might well differ from 0.5. Therefore, we suggest to use the age shift needed to restore equality of chance as mortality differential indicator. Precisely, for two remaining lifetimes such that $\mathrm{P}\left[T_{x}^{[1]} \leq T_{x}^{[2]}\right] \geq 0.5$, we define the age shift $\tau_{x}$ as the time lived under the second lifetime to restore equal survival chances, that is, as the root of the equation

$$
\mathrm{P}\left[T_{x}^{[1]} \leq T_{x}^{[2]}-\tau \mid T_{x}^{[2]}>\tau\right]=0.5 .
$$

Considering the evolution of $\tau_{x}$ as a function of attained age $x$ is particularly instructive to detect whether mortality gaps tend to accentuate with age, or not. 
Henceforth, we use a superscript "[k]" to indicate quantities relating to $T_{x}^{[k]}, k \in\{1,2\}$, such as

$$
\begin{aligned}
{ }_{t} q_{x}^{[k]} & =\mathrm{P}\left[T_{x}^{[k]} \leq t\right]=1-{ }_{t} p_{x}^{[k]} \\
\mu_{x+t}^{[k]} & =-\frac{\partial}{\partial t} \ln _{t} p_{x}^{[k]} .
\end{aligned}
$$

\subsection{Independent case}

In this section, we assume that the remaining lifetimes under consideration are mutually independent. This hypothesis may be regarded as being reasonable for two individuals in different social classes in a periodic setting, for instance. Denoting as $\omega$ the ultimate age for both lifetimes under consideration, possibly infinite, the probability involved in (2.1) can be written as

$$
\mathrm{P}\left[T_{x}^{[1]} \leq T_{x}^{[2]}-\tau \mid T_{x}^{[2]}>\tau\right]=1-\int_{0}^{\omega-x-\tau}{ }_{t}{ }_{x+\tau}^{[2]} \mu_{x+\tau+t}^{[2]} p_{x}^{[1]} \mathrm{d} t
$$

A grid search can be performed to identify the integer part of $\tau_{x}$. Its fractional part can then be obtained by interpolation.

\subsection{Conditionally independent case}

In this section, we assume that $T_{x}^{[1]}$ and $T_{x}^{[2]}$ are only conditionally independent, given a set of time factors. This corresponds to the case where $T_{x}^{[1]}$ and $T_{x}^{[2]}$ obey some mortality projection model depending on future trajectory of time factors $\boldsymbol{\kappa}^{[1]}$ and $\boldsymbol{\kappa}^{[2]}$, respectively. For instance, $T_{x}^{[1]}$ and $T_{x}^{[2]}$ may refer to male and female lifetimes in a given country and $\boldsymbol{\kappa}^{[1]}$ and $\boldsymbol{\kappa}^{[2]}$ represent the gender-specific time factors in a bivariate Lee-Carter model. Or $T_{x}^{[1]}$ and $T_{x}^{[2]}$ may relate to lifetimes in two different countries in a multi-population model, or to different generations.

The formula in the preceding section can then be applied conditional to $\left(\boldsymbol{\kappa}^{[1]}, \boldsymbol{\kappa}^{[2]}\right)$. In a periodic setting, a limited number of time factors enter the calculation (just two in the LeeCarter case, for instance) and the integrals can easily be evaluated numerically. Calculations are more difficult when cohorts are followed over time since whole trajectories of $\left(\boldsymbol{\kappa}^{[1]}, \boldsymbol{\kappa}^{[2]}\right)$ enter the calculation, resulting in a large number of conditioning variables in the probability 2.1. Simulation can then be conducted to obtain the age shift $\tau_{x}$. A first approximation of $\tau_{x}$, say $\tau_{x}^{[0]}$, can be obtained by replacing the time factors $\left(\boldsymbol{\kappa}^{[1]}, \boldsymbol{\kappa}^{[2]}\right)$ with their expected values. We can then simulate a large number of pairs $\left(T_{x}^{[1]}, T_{x}^{[2]}\right)$ by first generating a trajectory of future $\left(\boldsymbol{\kappa}^{[1]}, \boldsymbol{\kappa}^{[2]}\right)$ and then simulate remaining lifetimes according to the life tables corresponding to these time factors values. The probability in (2.1) can be approached by the corresponding proportion and the value of $\tau_{x}$ can be obtained by a grid search for values close to $\tau_{x}^{[0]}$. 


\section{$3 \quad$ Numerical illustrations}

\subsection{Mortality according to household total income in France}

The French National Institute of Statistics INSEE has published several studies of mortality by social class, educational levels and living standards. In this section, we consider the life tables produced by Blanpain (2018) based on the French permanent demographic sample (EDP), which incorporates tax data. Households are divided in 20 classes according to the quantiles of their total income over 2012-2016.

Consider for $T_{x}^{[1]}$ a remaining lifetime subject to the mortality for the poorest $5 \%$ of the population and for $T_{x}^{[2]}$ a remaining lifetime subject to the mortality for the richest $5 \%$ of the population. Here, we assume that $T_{x}^{[1]}$ and $T_{x}^{[2]}$ are mutually independent. We then get $\mathrm{P}\left[T_{x}^{[1]} \leq T_{x}^{[2]}\right]>0.5$ for all ages $x$ and both males and females. Figure 3.1 displays the age shifts $\tau_{x}$ restoring equivalence between these two groups for attained ages $x$ from birth (entire lifetime) to 90 . We can see there that the age shifts are pretty large and decrease with age. Life expectancies at birth in these two groups are 71.7 and 84.4 for males and 80.0 and 88.3 for females. The age shifts at birth equal to 11.7 years for males and 6.3 years for females thus compare with the differences of these life expectancies. We see that age shifts remain stable until age 30 , because of the low mortality risk at these ages, but start to drop at older ages. Expected remaining lifetimes at age 65 in these two groups are 15.8 and 21.8 for males and 20.4 and 25.0 for females, of the same order of age shifts equal to 6.8 years and 4.4 years, respectively. Contrarily to differences in life expectancies, the meaning of age shifts is clearer as they represent the comparative advantage of the higher socio-economic group if equality of survival chance is regarded as a meaningful objective.

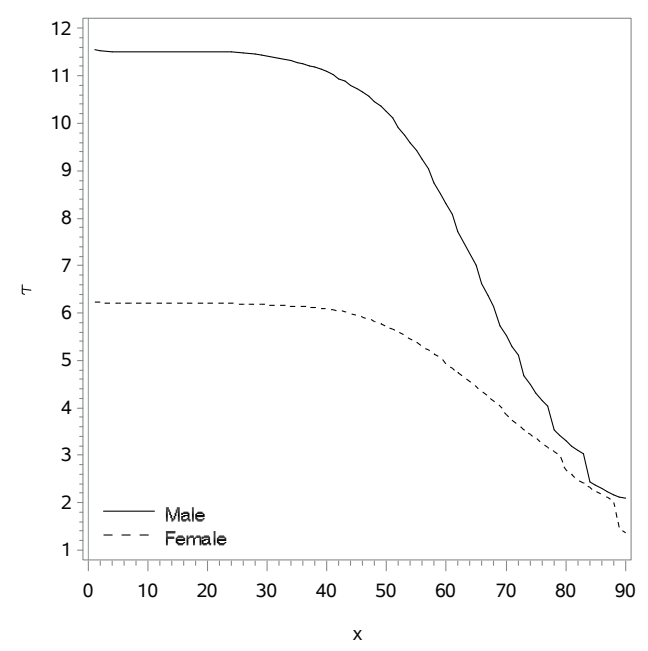

Figure 3.1: Age shifts $\tau_{x}$ for $x \in\{0,1, \ldots, 90\}$ solving equation $(2.1)$ when $T_{x}^{[1]}$ correspond to a lifetime subject to the mortality for the poorest $5 \%$ and $T_{x}^{[2]}$ to a lifetime subject to the mortality for the richest $5 \%$ of the French population. Values obtained from the life tables given in Blanpain (2018) by assuming mutual independence. 


\subsection{Mortality projection for Belgium}

In this section, we consider mortality statistics for Belgium, general population. Observed mortality rates and corresponding exposures are available from https://www.plan.be/ databases/. Here, we consider calendar years 1991 to 2018 and ages 30 to 109. Future mortality is projected using the models of Cadena and Denuit (2016), henceforth referred to as "CD" and of Lee and Carter (1992), henceforth referred to as "LC". Package StMoMo is used for applying the LC method and the function auto.arima in package forecast to forecast time series. Both packages are available in the $\mathrm{R}$ programming environment.

We follow generations over time so that the lifetimes under consideration are only conditionally independent, given the time factor involved in the CD or LC model. Precisely, we consider individuals aged $x \in\{30,31, \ldots, 90\}$ in calendar year $r, r \in\{1992,2005\}$, and we compare their survival with individuals reaching the same age $x$ in calendar year $r+13$. The results are displayed in Figure 3.2. The upper panels display the approximations $\tau_{x}^{[0]}$ obtained by replacing the time factors with their expected values whereas the age shifts appearing in the lower panels have been obtained by simulation.

We see there that the approximations $\tau_{x}^{[0]}$ are very close to the true values $\tau_{x}$ solving equation (2.1). It is also visible that the choice of the mortality projection model impacts on the age shifts restoring equality of survival chance, except for the middle ages 60-80 where both models produce similar $\tau_{x}$ values. Considering the magnitude of age shifts as a measure of mortality improvement, we see that CD forecasts more progress at younger ages (before 50) and at older ages, compared to LC. The graphs in Figure 3.2 thus help to assess the longevity dynamics across ages.

\section{Bibliography}

- Arcones, M., Kvam, P., Samaniego, F.J. (2002). On nonparametric estimation of distributions subject to a stochastic precedence constraint. Journal of the American Statistical Association 97, 170-182.

- Blanpain, N. (2018). L'espérance de vie par niveau de vie. Méthode et principaux résultats. INSEE, Document de Travail F1801. Available from http://www.epsilon.insee.fr/

- Boland, P.J., Singh, H., Cukic, B. (2004). The stochastic precedence ordering with applications in sampling and testing. Journal of Applied Probability 41, 73-82.

- Brown, G.G., Rutemiller, H.C. (1973). Evaluation of $\operatorname{Pr}\{X \geq Y\}$ when both $X$ and $Y$ are from three-parameter Weibull distributions. IEEE Transactions on Reliability $22,78-82$.

- Cadena, M., Denuit, M. (2016). Semi-parametric accelerated hazard relational models with applications to mortality projections. Insurance: Mathematics and Economics $68,1-16$.

- De Santis, E., Fantozzi, F., Spizzichino, F. (2015). Relations between stochastic orderings and generalized stochastic precedence. Probability in the Engineering and Informational Sciences 29, 329-343. 

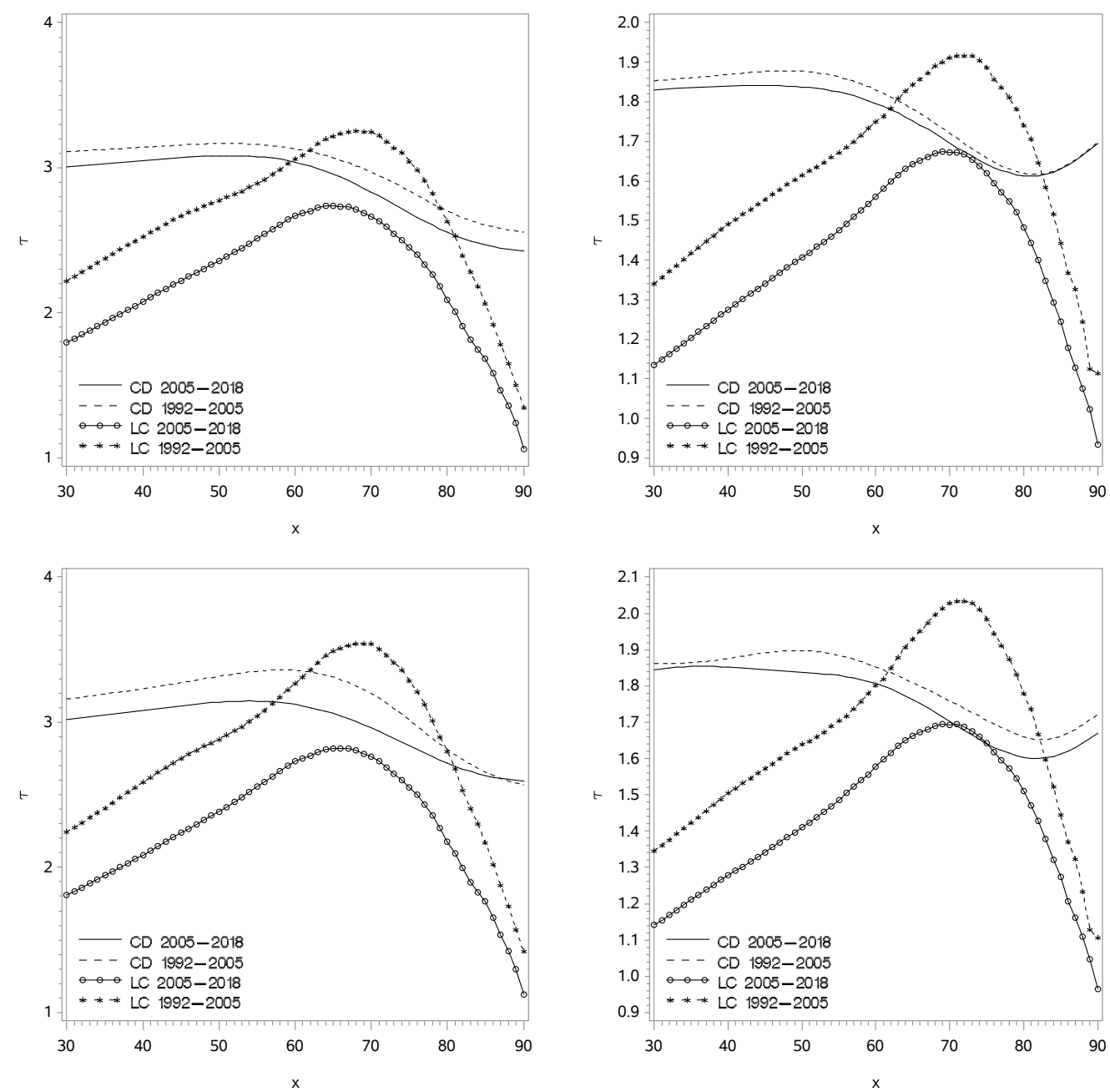

Figure 3.2: Age shifts $\tau_{x}$ for $\left.x \in\{30,31, \ldots, 90\}\right)$ solving equation (2.1) when $T_{x}^{[1]}$ correspond to a lifetime subject to the mortality for generation aged $x$ in calendar year $r$, $r \in\{1992,2005\}$, and $T_{x}^{[2]}$ to a lifetime subject to the mortality for generation aged $x$ in calendar year $r+13$, Belgian general population. Initial approximations $\tau_{x}^{[0]}$ are displayed in the top panels, solutions $\tau_{x}$ to (2.1) obtained by simulation are displayed in the bottom panels. Males appear on the left, females on the right.

- Finkelstein, M. (2013). On some comparisons of lifetimes for reliability analysis. Reliability Engineering and System Safety 119, 300-304.

- Lee, R.D., Carter, L. (1992). Modelling and forecasting the time series of US mortality. Journal of the American Statistical Association 87, 659-671.

- Navarro, J., Rubio, R. (2010). Comparisons of coherent systems using stochastic precedence. TEST 19, 469-486. 